\title{
Beyond the Gaussian
}

Kazuyuki FUJII

Department of Mathematical Sciences, Yokohama City University, Yokohama, 236-0027 Japan E-mail: fujii@yokohama-cu.ac.jp

Received January 12, 2011, in final form February 28, 2011; Published online March 04, 2011

doi:10.3842/SIGMA.2011.022

\begin{abstract}
In this paper we present a non-Gaussian integral based on a cubic polynomial, instead of a quadratic, and give a fundamental formula in terms of its discriminant. It gives a mathematical reinforcement to the recent result by Morozov and Shakirov. We also present some related results. This is simply one modest step to go beyond the Gaussian but it already reveals many obstacles related with the big challenge of going further beyond the Gaussian.
\end{abstract}

Key words: non-Gaussian integral; renormalized integral; discriminant; cubic equation

2010 Mathematics Subject Classification: 11D25; 11R29; 26B20; 81Q99

\section{Introduction}

The Gaussian is an abbreviation of all subjects related to the Gauss function $e^{-\left(p x^{2}+q x+r\right)}$ like the Gaussian beam, Gaussian process, Gaussian noise, etc. It plays a fundamental role in mathematics, statistics, physics and related disciplines. It is generally conceived that any attempts to generalize the Gaussian results would meet formidable difficulties. Hoping to overcome this high wall of difficulties of going beyond the Gaussian in the near future, a first step was introduced in [1]. This paper is its polished version.

In the paper [2] the following "formula" is reported:

$$
\iint e^{-\left(a x^{3}+b x^{2} y+c x y^{2}+d y^{3}\right)} d x d y=\frac{1}{\sqrt[6]{-D}},
$$

where $D$ is the discriminant of the cubic equation

$$
a x^{3}+b x^{2}+c x+d=0,
$$

and it is given by

$$
D=b^{2} c^{2}+18 a b c d-4 a c^{3}-4 b^{3} d-27 a^{2} d^{2} .
$$

The formula (1) is of course non-Gaussian. However, if we consider it in the framework of the real category then (1) is not correct because the left hand side diverges. In this paper we treat only the real category, and so $a, b, c, d, x, y$ are real numbers.

Formally, by performing the change of variable $x=t \rho, y=\rho$ for (1) we have

$$
\text { 1.h.s. of } \begin{aligned}
(1) & =\iint e^{-\rho^{3}\left(a t^{3}+b t^{2}+c t+d\right)}|\rho| d t d \rho=\int\left\{\int e^{-\left(a t^{3}+b t^{2}+c t+d\right) \rho^{3}}|\rho| d \rho\right\} d t \\
& =\int|\sigma| e^{-\sigma^{3}} d \sigma \int \frac{1}{\left|\sqrt[3]{\left(a t^{3}+b t^{2}+c t+d\right)}\right| \sqrt[3]{\left(a t^{3}+b t^{2}+c t+d\right)}} d t
\end{aligned}
$$

by the change of variable $\sigma=\sqrt[3]{a t^{3}+b t^{2}+c t+d} \rho$. 
The divergence comes from

$$
\int|\sigma| e^{-\sigma^{3}} d \sigma
$$

while the main part is

$$
\int \frac{1}{\left|\sqrt[3]{\left(a x^{3}+b x^{2}+c x+d\right)}\right| \sqrt[3]{\left(a x^{3}+b x^{2}+c x+d\right)}} d x
$$

under the change $t \rightarrow x$. As a kind of renormalization the integral may be defined like

$$
\ddagger \iint_{\mathbb{R}^{2}} e^{-\left(a x^{3}+b x^{2} y+c x y^{2}+d y^{3}\right)} d x d y \ddagger=\int_{\mathbb{R}} \frac{1}{\left|\sqrt[3]{\left(a x^{3}+b x^{2}+c x+d\right)}\right| \sqrt[3]{\left(a x^{3}+b x^{2}+c x+d\right)}} d x .
$$

However, the right hand side lacks proper symmetry. If we set

$$
F(a, b, c, d)=\iint_{D_{R}} e^{-\left(a x^{3}+b x^{2} y+c x y^{2}+d y^{3}\right)} d x d y,
$$

where $D_{R}=[-R, R] \times[-R, R]$, then it is easy to see

$$
F(-a,-b,-c,-d)=F(a, b, c, d) .
$$

Namely, $F$ is invariant under $\mathbb{Z}_{2}$-action. This symmetry is important and must be kept even in the renormalization process. The right hand side in the "definition" above is clearly not invariant. Therefore, by modifying it slightly we reach the renormalized integral

\section{Definition 1.}

$$
\ddagger \iint_{\mathbb{R}^{2}} e^{-\left(a x^{3}+b x^{2} y+c x y^{2}+d y^{3}\right)} d x d y \ddagger=\int_{\mathbb{R}} \frac{1}{\sqrt[3]{\left(a x^{3}+b x^{2}+c x+d\right)^{2}}} d x .
$$

We believe that the definition is not so bad (see the Section 4).

In the paper we calculate the right hand side of (3) directly, which will give some interesting results and a new perspective. The result gives a mathematical reinforcement to the result [2] by Morozov and Shakirov.

\section{Main result}

Before stating the result let us make some preparations. The Gamma function $\Gamma(p)$ is defined by

$$
\Gamma(p)=\int_{0}^{\infty} e^{-x} x^{p-1} d x \quad(p>0)
$$

and the Beta function $B(p, q)$ is

$$
B(p, q)=\int_{0}^{1} x^{p-1}(1-x)^{q-1} d x \quad(p, q>0) .
$$

Note that the Beta function is rewritten as

$$
B(p, q)=\int_{0}^{\infty} \frac{x^{p-1}}{(1+x)^{p+q}} d x .
$$

See [3] for more detail. Now we are in a position to state the result. 


\section{Fundamental formula.}

(I) For $D<0$

$$
\int_{\mathbb{R}} \frac{1}{\sqrt[3]{\left(a x^{3}+b x^{2}+c x+d\right)^{2}}} d x=\frac{C_{-}}{\sqrt[6]{-D}}
$$

where

$$
C_{-}=\sqrt[3]{2} B\left(\frac{1}{2}, \frac{1}{6}\right)
$$

(II) For $D>0$

$$
\int_{\mathbb{R}} \frac{1}{\sqrt[3]{\left(a x^{3}+b x^{2}+c x+d\right)^{2}}} d x=\frac{C_{+}}{\sqrt[6]{D}},
$$

where

$$
C_{+}=3 B\left(\frac{1}{3}, \frac{1}{3}\right) .
$$

(III) $C_{-}$and $C_{+}$are related by $C_{+}=\sqrt{3} C_{-}$through the identity

$$
\sqrt{3} B\left(\frac{1}{3}, \frac{1}{3}\right)=\sqrt[3]{2} B\left(\frac{1}{2}, \frac{1}{6}\right)
$$

Our result shows that the integral depends on the sign of $D$, and so our question is as follows. Problem. Can the result be derived from the method developed in [2]?

A comment is in order. If we treat the Gaussian case $\left(e^{-\left(a x^{2}+b x y+c y^{2}\right)}\right)$ then the integral is reduced to

$$
\int_{\mathbb{R}} \frac{1}{a x^{2}+b x+c} d x=\frac{2 \pi}{\sqrt{-D}}
$$

if $a>0$ and $D=b^{2}-4 a c<0$. Noting

$$
\pi=\frac{\sqrt{\pi} \sqrt{\pi}}{1}=\frac{\Gamma\left(\frac{1}{2}\right) \Gamma\left(\frac{1}{2}\right)}{\Gamma(1)}=B\left(\frac{1}{2}, \frac{1}{2}\right)
$$

(8) should be read as

$$
\int_{\mathbb{R}} \frac{1}{a x^{2}+b x+c} d x=\frac{2 B\left(\frac{1}{2}, \frac{1}{2}\right)}{\sqrt{-D}} .
$$

\section{Proof of the formula}

The proof is delicate. In order to prevent possible misunderstanding we present a detailed proof in this section.

Proof of (I). We prove (5) in case of $D<0$.

First we consider the special case where $a=0$ in the cubic equation $a x^{3}+b x^{2}+c x+d$. Namely, we calculate the integral

$$
\int_{\mathbb{R}} \frac{1}{\sqrt[3]{\left(b x^{2}+c x+d\right)^{2}}} d x .
$$


Noting $-D=b^{2}\left(4 b d-c^{2}\right)>0$ we obtain

$$
\begin{aligned}
\int_{\mathbb{R}} & \frac{1}{\sqrt[3]{\left(b x^{2}+c x+d\right)^{2}}} d x=\int_{\mathbb{R}} \frac{1}{\sqrt[3]{b^{2}} \sqrt[3]{\left(x^{2}+\frac{c}{b} x+\frac{d}{b}\right)^{2}}} d x \\
& =\frac{1}{\sqrt[3]{b^{2}}} \int_{\mathbb{R}} \frac{1}{\sqrt[3]{\left(\left(x+\frac{c}{2 b}\right)^{2}+\frac{d}{b}-\frac{c^{2}}{4 b^{2}}\right)^{2}}} d x=\frac{1}{\sqrt[3]{b^{2}}} \int_{\mathbb{R}} \frac{1}{\sqrt[3]{\left(x^{2}+\frac{4 b d-c^{2}}{4 b^{2}}\right)^{2}}} d x \\
T^{2} \equiv & \frac{\frac{4 b d-c^{2}}{\underline{\underline{4 k}}}>0}{=} \frac{1}{\sqrt[3]{b^{2}}} \int_{\mathbb{R}} \frac{1}{\sqrt[3]{\left(x^{2}+T^{2}\right)^{2}}} d x \stackrel{x=T y}{=} \frac{1}{\sqrt[3]{b^{2}}} \int_{\mathbb{R}} \frac{T}{\sqrt[3]{T^{4}} \sqrt[3]{\left(y^{2}+1\right)^{2}}} d y \\
= & \frac{2}{\sqrt[3]{b^{2} T}} \int_{0}^{\infty} \frac{1}{\sqrt[3]{\left(y^{2}+1\right)^{2}}} d y \stackrel{y=\sqrt{x}}{=} \frac{2}{\sqrt[3]{b^{2} T}} \int_{0}^{\infty} \frac{1}{\sqrt[3]{(x+1)^{2}} \frac{d x}{2 \sqrt{x}}} \\
= & \frac{1}{\sqrt[3]{b^{2} T}} \int_{0}^{\infty} \frac{x^{-\frac{1}{2}}}{(x+1)^{\frac{2}{3}}} d x=\frac{B\left(\frac{1}{2}, \frac{1}{6}\right)}{\sqrt[3]{b^{2} T}}=\frac{B\left(\frac{1}{2}, \frac{1}{6}\right)}{\sqrt[6]{b^{4} T^{2}}}=\frac{\sqrt[3]{2} B\left(\frac{1}{2}, \frac{1}{6}\right)}{\sqrt[6]{b^{2}\left(4 b d-c^{2}\right)}}=\frac{\sqrt[3]{2} B\left(\frac{1}{2}, \frac{1}{6}\right)}{\sqrt[6]{-D}} .
\end{aligned}
$$

Now we consider the general case of $a \neq 0$. From the condition $D<0$ there is (only) one real root of the cubic equation $a x^{3}+b x^{2}+c x+d=0$. Let us denote it by $\alpha$. From the equation

$$
a x^{3}+b x^{2}+c x+d=(x-\alpha)\left(a x^{2}+k x+l\right), \quad a \alpha^{3}+b \alpha^{2}+c \alpha+d=0
$$

we have easily

$$
b=k-a \alpha, \quad c=l-k \alpha, \quad d=-l \alpha .
$$

First we assume $\alpha=0$. In this case $d=0$ and

$$
a x^{3}+b x^{2}+c x+d=x\left(a x^{2}+b x+c\right) .
$$

Then

$$
\begin{aligned}
& \int_{\mathbb{R}} \frac{1}{\sqrt[3]{x^{2}\left(a x^{2}+b x+c\right)^{2}}} d x=\int_{0}^{\infty} \frac{1}{\sqrt[3]{x^{2}\left(a x^{2}+b x+c\right)^{2}}} d x+\int_{-\infty}^{0} \frac{1}{\sqrt[3]{x^{2}\left(a x^{2}+b x+c\right)^{2}}} d x \\
&\left.\stackrel{x=\frac{1}{y}}{=} \int_{\infty}^{0} \frac{1}{\sqrt[3]{\frac{1}{y^{2}}\left(\frac{a}{y^{2}}+\frac{b}{y}+c\right)^{2}}}\left(-\frac{d y}{y^{2}}\right)+\int_{0}^{-\infty} \frac{1 y}{y^{2}}\right) \\
& \quad=\int_{0}^{\infty} \frac{1}{\sqrt[3]{\frac{1}{y^{2}}\left(\frac{a}{y^{2}}+\frac{b}{y}+c\right)^{2}}} d y+\int_{-\infty}^{0} \frac{1}{\sqrt[3]{\left.\left(c y^{2}+b y+a\right)^{2}+b y+a\right)^{2}}} d y \\
&=\int_{\mathbb{R}} \frac{1}{\sqrt[3]{\left(c y^{2}+b y+a\right)^{2}}} d y \stackrel{(b \rightarrow c ; c \rightarrow b ; d \rightarrow a)}{=} \frac{\sqrt[3]{2} B\left(\frac{1}{2}, \frac{1}{6}\right)}{\sqrt[6]{c^{2}\left(4 a c-b^{2}\right)}}=\frac{\sqrt[3]{2} B\left(\frac{1}{2}, \frac{1}{6}\right)}{\sqrt[6]{-D}} .
\end{aligned}
$$

Next, let us calculate the case $\alpha \neq 0$ :

$$
\begin{aligned}
\int_{\mathbb{R}} & \frac{1}{\sqrt[3]{(x-\alpha)^{2}\left(a x^{2}+k x+l\right)^{2}}} d x \stackrel{x=\underline{y}+\alpha}{=} \int_{\mathbb{R}} \frac{1}{\sqrt[3]{y^{2}\left\{a(y+\alpha)^{2}+k(y+\alpha)+l\right\}^{2}}} d y \\
& =\int_{\mathbb{R}} \frac{1}{\sqrt[3]{y^{2}\left\{a y^{2}+(2 a \alpha+k) y+\left(a \alpha^{2}+k \alpha+l\right)\right\}^{2}}} d y \\
& \stackrel{(10))}{=} \frac{\sqrt[3]{2} B\left(\frac{1}{2}, \frac{1}{6}\right)}{\sqrt[6]{\left(a \alpha^{2}+k \alpha+l\right)^{2}\left\{4 a\left(a \alpha^{2}+k \alpha+l\right)-(2 a \alpha+k)^{2}\right\}}} \\
= & \frac{\sqrt[3]{2} B\left(\frac{1}{2}, \frac{1}{6}\right)}{\sqrt[6]{\left(a \alpha^{2}+k \alpha+l\right)^{2}\left(4 a l-k^{2}\right)}} .
\end{aligned}
$$


Key Lemma. From (10) the following equation holds

$$
\left(a \alpha^{2}+k \alpha+l\right)^{2}\left(4 a l-k^{2}\right)=27 a^{2} d^{2}+4 a c^{3}-18 a b c d-b^{2} c^{2}+4 b^{3} d=-D .
$$

The proof is straightforward but tedious.

Therefore, from both (11) and (12) we obtain the formula

$$
\int_{\mathbb{R}} \frac{1}{\sqrt[3]{(x-\alpha)^{2}\left(a x^{2}+k x+l\right)^{2}}} d x=\frac{\sqrt[3]{2} B\left(\frac{1}{2}, \frac{1}{6}\right)}{\sqrt[6]{-D}} .
$$

Proof of (II). We prove (6) in case of $D>0$. Let us start with the evaluation of the following integral

$$
\int_{\mathbb{R}} \frac{1}{\sqrt[3]{x^{2}(x-\alpha)^{2}}} d x
$$

for $\alpha>0$. Then

$$
\begin{aligned}
\int_{\mathbb{R}} \frac{1}{\sqrt[3]{x^{2}(x-\alpha)^{2}}} d x & =\int_{-\infty}^{0} \frac{1}{\sqrt[3]{x^{2}(x-\alpha)^{2}}} d x+\int_{0}^{\infty} \frac{1}{\sqrt[3]{x^{2}(x-\alpha)^{2}}} d x \\
& =\int_{0}^{\infty} \frac{1}{\sqrt[3]{x^{2}(x+\alpha)^{2}}} d x+\int_{0}^{\infty} \frac{1}{\sqrt[3]{x^{2}(x-\alpha)^{2}}} d x
\end{aligned}
$$

where the change of variable $x \rightarrow-x$ for the first term of the right hand side was made.

Each term can be evaluated elementarily:

$$
\int_{0}^{\infty} \frac{1}{\sqrt[3]{x^{2}(x+\alpha)^{2}}} d x \stackrel{x=\alpha t}{=} \frac{1}{\sqrt[3]{\alpha}} \int_{0}^{\infty} \frac{1}{\sqrt[3]{t^{2}(t+1)^{2}}} d t=\alpha^{-\frac{1}{3}} \int_{0}^{\infty} \frac{t^{-\frac{2}{3}}}{(t+1)^{\frac{2}{3}}} d t=\alpha^{-\frac{1}{3}} B\left(\frac{1}{3}, \frac{1}{3}\right),
$$

while

$$
\begin{aligned}
\int_{0}^{\infty} \frac{1}{\sqrt[3]{x^{2}(x-\alpha)^{2}}} d x & \stackrel{x=\alpha t}{=} \alpha^{-\frac{1}{3}} \int_{0}^{\infty} \frac{1}{\sqrt[3]{t^{2}(t-1)^{2}}} d t \\
& =\alpha^{-\frac{1}{3}}\left\{\int_{0}^{1} \frac{1}{\sqrt[3]{t^{2}(t-1)^{2}}} d t+\int_{1}^{\infty} \frac{1}{\sqrt[3]{t^{2}(t-1)^{2}}} d t\right\} \\
& =\alpha^{-\frac{1}{3}}\left\{\int_{0}^{1} \frac{1}{\sqrt[3]{t^{2}(1-t)^{2}}} d t+\int_{1}^{\infty} \frac{1}{\sqrt[3]{t^{2}(t-1)^{2}}} d t\right\} \\
& =2 \alpha^{-\frac{1}{3}} \int_{0}^{1} \frac{1}{\sqrt[3]{t^{2}(1-t)^{2}}} d t=2 \alpha^{-\frac{1}{3}} \int_{0}^{1} t^{-\frac{2}{3}}(1-t)^{-\frac{2}{3}} d t \\
& =2 \alpha^{-\frac{1}{3}} B\left(\frac{1}{3}, \frac{1}{3}\right)
\end{aligned}
$$

where we have used

$$
\int_{1}^{\infty} \frac{1}{\sqrt[3]{t^{2}(t-1)^{2}}} d t \stackrel{t=\frac{1}{s}}{=} \int_{1}^{0} \frac{1}{\sqrt[3]{\frac{1}{s^{2}} \frac{(1-s)^{2}}{s^{2}}}}\left(-\frac{d s}{s^{2}}\right)=\int_{0}^{1} \frac{1}{\sqrt[3]{s^{2}(1-s)^{2}}} d s=\int_{0}^{1} \frac{1}{\sqrt[3]{t^{2}(1-t)^{2}}} d t .
$$

From (13) we have

$$
\int_{\mathbb{R}} \frac{1}{\sqrt[3]{x^{2}(x-\alpha)^{2}}} d x=3 \alpha^{-\frac{1}{3}} B\left(\frac{1}{3}, \frac{1}{3}\right)=\frac{3 B\left(\frac{1}{3}, \frac{1}{3}\right)}{\sqrt[3]{\alpha}} .
$$


Now we consider the special case $a=0$ in the cubic equation $a x^{3}+b x^{2}+c x+d$. Then by $D=b^{2}\left(c^{2}-4 b d\right)>0$ we obtain

$$
\begin{aligned}
& \int_{\mathbb{R}} \frac{1}{\sqrt[3]{\left(b x^{2}+c x+d\right)^{2}}} d x=\int_{-\infty}^{\infty} \frac{1}{\sqrt[3]{\left\{b\left(x+\frac{c}{2 b}\right)^{2}-\frac{c^{2}-4 b d}{4 b}\right\}^{2}}} d x=\int_{-\infty}^{\infty} \frac{1}{\sqrt[3]{\left(b x^{2}-\frac{c^{2}-4 b d}{4 b}\right)^{2}}} d x \\
& \alpha^{2}=\frac{c^{2}-4 b d}{\underline{\underline{4 b^{2}}}}>0 \frac{1}{\sqrt[3]{b^{2}}} \int_{-\infty}^{\infty} \frac{1}{\sqrt[3]{\left(x^{2}-\alpha^{2}\right)^{2}}} d x=\frac{1}{\sqrt[3]{b^{2}}} \int_{-\infty}^{\infty} \frac{1}{\sqrt[3]{(x-\alpha)^{2}(x+\alpha)^{2}}} d x \\
& y=\stackrel{x+\alpha}{=} \frac{1}{\sqrt[3]{b^{2}}} \int_{-\infty}^{\infty} \frac{1}{\sqrt[3]{y^{2}(y-2 \alpha)^{2}}} d y \stackrel{(13)}{=} \frac{1}{\sqrt[3]{b^{2}}} \frac{3 B\left(\frac{1}{3}, \frac{1}{3}\right)}{\sqrt[3]{2 \alpha}} \\
& =\frac{3 B\left(\frac{1}{3}, \frac{1}{3}\right)}{\sqrt[3]{2 \alpha b^{2}}}=\frac{3 B\left(\frac{1}{3}, \frac{1}{3}\right)}{\sqrt[6]{4 \alpha^{2} b^{4}}}=\frac{3 B\left(\frac{1}{3}, \frac{1}{3}\right)}{\sqrt[6]{b^{2}\left(c^{2}-4 b d\right)}}=\frac{3 B\left(\frac{1}{3}, \frac{1}{3}\right)}{\sqrt[6]{D}} .
\end{aligned}
$$

Next we consider the remaining general case of $a \neq 0$. From the condition $D>0$ there are three real solutions in the equation $a x^{3}+b x^{2}+c x+d=0$. We denote one of them by $\alpha$. Remember the relations $b=k-a \alpha, c=l-k \alpha, d=-l \alpha$ from the equation

$$
a x^{3}+b x^{2}+c x+d=(x-\alpha)\left(a x^{2}+k x+l\right), \quad a \alpha^{3}+b \alpha^{2}+c \alpha+d=0 .
$$

First we assume $\alpha=0$. Then

$$
a x^{3}+b x^{2}+c x+d=x\left(a x^{2}+b x+c\right)
$$

and from $D=c^{2}\left(b^{2}-4 a c\right)$ we have

$$
\begin{gathered}
\int_{\mathbb{R}} \frac{1}{\sqrt[3]{x^{2}\left(a x^{2}+b x+c\right)^{2}}} d x=\int_{0}^{\infty} \frac{1}{\sqrt[3]{x^{2}\left(a x^{2}+b x+c\right)^{2}}} d x+\int_{-\infty}^{0} \frac{1}{\sqrt[3]{x^{2}\left(a x^{2}+b x+c\right)^{2}}} d x \\
\stackrel{x=\frac{1}{y}}{=} \int_{\infty}^{0} \frac{1}{\sqrt[3]{\frac{1}{y^{2}}\left(\frac{a}{y^{2}}+\frac{b}{y}+c\right)^{2}}}\left(-\frac{d y}{y^{2}}\right)+\int_{0}^{-\infty} \frac{1}{\sqrt[3]{\frac{1}{y^{2}}\left(\frac{a}{y^{2}}+\frac{b}{y}+c\right)^{2}}} \\
\quad=\int_{0}^{\infty} \frac{1}{\sqrt[3]{\left(c y^{2}+b y+a\right)^{2}}} d y+\int_{-\infty}^{0} \frac{1}{\sqrt[3]{\left(c y^{2}+b y+a\right)^{2}}} d y=\int_{\mathbb{R}} \frac{1}{\sqrt[3]{\left(c y^{2}+b y+a\right)^{2}}} d y \\
\text { (14) }(c \rightarrow b ; b \rightarrow c ; a \rightarrow d) \frac{3 B\left(\frac{1}{3}, \frac{1}{3}\right)}{=}=\frac{3 B\left(\frac{1}{3}, \frac{1}{3}\right)}{\sqrt[6]{c^{2}\left(b^{2}-4 a c\right)}}
\end{gathered}
$$

For the case $\alpha \neq 0$ we obtain the formula

$$
\begin{aligned}
\int_{\mathbb{R}} & \frac{1}{\sqrt[3]{(x-\alpha)^{2}\left(a x^{2}+k x+l\right)^{2}}} d x \stackrel{x=\underline{y}+\alpha}{=} \int_{\mathbb{R}} \frac{1}{\sqrt[3]{y^{2}\left\{a(y+\alpha)^{2}+k(y+\alpha)+l\right\}^{2}}} d y \\
& =\int_{\mathbb{R}} \frac{1}{\sqrt[3]{y^{2}\left\{a y^{2}+(2 a \alpha+k) y+\left(a \alpha^{2}+k \alpha+l\right)\right\}^{2}}} d y \\
& \stackrel{(15)}{=} \frac{3 B\left(\frac{1}{3}, \frac{1}{3}\right)}{\sqrt[6]{\left(a \alpha^{2}+k \alpha+l\right)^{2}\left\{(2 a \alpha+k)^{2}-4 a\left(a \alpha^{2}+k \alpha+l\right)\right\}}} \\
& =\frac{3 B\left(\frac{1}{3}, \frac{1}{3}\right)}{\sqrt[6]{\left(a \alpha^{2}+k \alpha+l\right)^{2}\left(k^{2}-4 a l\right)}} \stackrel{(12)}{=} \frac{3 B\left(\frac{1}{3}, \frac{1}{3}\right)}{\sqrt[6]{D}} .
\end{aligned}
$$

Proof of (III). We prove the relation (7). Let us make some preparations. For the Gamma function (4) there are well-known formulas (see for example [3])

$$
B(x, y)=\frac{\Gamma(x) \Gamma(y)}{\Gamma(x+y)} \quad(x, y>0)
$$




$$
\begin{aligned}
& \Gamma(x) \Gamma(1-x)=\frac{\pi}{\sin (\pi x)} \quad(0<x<1), \\
& \Gamma\left(\frac{x}{2}\right) \Gamma\left(\frac{x+1}{2}\right)=\frac{\sqrt{\pi}}{2^{x-1}} \Gamma(x)=2^{1-x} \Gamma\left(\frac{1}{2}\right) \Gamma(x) .
\end{aligned}
$$

(18) is called the Legendre's relation. In the formula we set $x=2 / 3$, then

$$
\Gamma\left(\frac{1}{3}\right) \Gamma\left(\frac{5}{6}\right)=\sqrt[3]{2} \Gamma\left(\frac{1}{2}\right) \Gamma\left(\frac{2}{3}\right)
$$

Multiplying both sides by $\Gamma(1 / 6)$ gives

$$
\begin{aligned}
\Gamma\left(\frac{1}{3}\right) \Gamma\left(\frac{5}{6}\right) \Gamma\left(\frac{1}{6}\right)=\sqrt[3]{2} \Gamma\left(\frac{1}{2}\right) \Gamma\left(\frac{2}{3}\right) \Gamma\left(\frac{1}{6}\right) \\
\quad \Longleftrightarrow \quad \Gamma\left(\frac{1}{3}\right) \frac{\pi}{\sin \left(\frac{\pi}{6}\right)}=\sqrt[3]{2} \Gamma\left(\frac{1}{2}\right) \Gamma\left(\frac{1}{6}\right) \Gamma\left(\frac{2}{3}\right) \\
\quad \Longleftrightarrow \quad 2 \pi \Gamma\left(\frac{1}{3}\right)=\sqrt[3]{2} \Gamma\left(\frac{1}{2}\right) \Gamma\left(\frac{1}{6}\right) \Gamma\left(\frac{2}{3}\right) \\
\quad \Longleftrightarrow \quad 2 \pi \frac{\Gamma\left(\frac{1}{3}\right)}{\Gamma\left(\frac{2}{3}\right)^{2}}=\sqrt[3]{2} \frac{\Gamma\left(\frac{1}{2}\right) \Gamma\left(\frac{1}{6}\right)}{\Gamma\left(\frac{2}{3}\right)} \\
\quad \Longleftrightarrow \quad 2 \pi \frac{\Gamma\left(\frac{1}{3}\right) \Gamma\left(\frac{1}{3}\right)}{\Gamma\left(\frac{2}{3}\right) \Gamma\left(\frac{1}{3}\right) \Gamma\left(\frac{2}{3}\right)}=\sqrt[3]{2} B\left(\frac{1}{2}, \frac{1}{6}\right) \\
\quad \Longleftrightarrow \quad 2 \pi \frac{\Gamma\left(\frac{1}{3}\right)^{2}}{\frac{\pi}{\sin \left(\frac{\pi}{3}\right)} \Gamma\left(\frac{2}{3}\right)}=\sqrt[3]{2} B\left(\frac{1}{2}, \frac{1}{6}\right) \\
\quad \Longleftrightarrow \quad \sqrt{3} B\left(\frac{1}{3}, \frac{1}{3}\right)=\sqrt[3]{2} B\left(\frac{1}{2}, \frac{1}{6}\right)
\end{aligned}
$$

where we have used formulas (16) and (17) several times.

The proof of (7) is now complete.

\section{Renormalized integral revisited}

In this section let us check whether the renormalized integral (3) is reasonable or not by making use of the results in the Section 3.

In the introduction we introduced the following integral defined on $D_{R}=[-R, R] \times[-R, R]$

$$
F(a, b, c, d)=\iint_{D_{R}} e^{-\left(a x^{3}+b x^{2} y+c x y^{2}+d y^{3}\right)} d x d y .
$$

For this it is easy to see

$$
\begin{aligned}
& \left(\frac{\partial}{\partial a} \frac{\partial}{\partial d}-\frac{\partial}{\partial b} \frac{\partial}{\partial c}\right) F(a, b, c, d) \\
& \quad=\iint_{D_{R}}\left(x^{3} \cdot y^{3}-x^{2} y \cdot x y^{2}\right) e^{-\left(a x^{3}+b x^{2} y+c x y^{2}+d y^{3}\right)} d x d y=0, \\
& \left(\frac{\partial}{\partial b} \frac{\partial}{\partial b}-\frac{\partial}{\partial a} \frac{\partial}{\partial c}\right) F(a, b, c, d) \\
& \quad=\iint_{D_{R}}\left(x^{2} y \cdot x^{2} y-x^{3} \cdot x y^{2}\right) e^{-\left(a x^{3}+b x^{2} y+c x y^{2}+d y^{3}\right)} d x d y=0,
\end{aligned}
$$




$$
\begin{aligned}
& \left(\frac{\partial}{\partial c} \frac{\partial}{\partial c}-\frac{\partial}{\partial b} \frac{\partial}{\partial d}\right) F(a, b, c, d) \\
& \quad=\iint_{D_{R}}\left(x y^{2} \cdot x y^{2}-x^{2} y \cdot y^{3}\right) e^{-\left(a x^{3}+b x^{2} y+c x y^{2}+d y^{3}\right)} d x d y=0 .
\end{aligned}
$$

On the other hand, if we set

$$
\mathcal{F}(a, b, c, d)=\int_{\mathbb{R}} \frac{1}{\sqrt[3]{\left(a x^{3}+b x^{2}+c x+d\right)^{2}}} d x=\frac{C_{ \pm}}{\sqrt[6]{ \pm D}}
$$

then we can also verify the same relations:

$$
\begin{aligned}
& \left(\frac{\partial}{\partial a} \frac{\partial}{\partial d}-\frac{\partial}{\partial b} \frac{\partial}{\partial c}\right) \mathcal{F}(a, b, c, d)=0, \quad\left(\frac{\partial}{\partial b} \frac{\partial}{\partial b}-\frac{\partial}{\partial a} \frac{\partial}{\partial c}\right) \mathcal{F}(a, b, c, d)=0 \\
& \left(\frac{\partial}{\partial c} \frac{\partial}{\partial c}-\frac{\partial}{\partial b} \frac{\partial}{\partial d}\right) \mathcal{F}(a, b, c, d)=0 .
\end{aligned}
$$

Verification by hand is rather tough, but it can be done easily by use of MATHEMATICA ${ }^{1}$.

From (19) and (20) we can conclude that our renormalized integral (3) is reasonable enough.

\section{Discriminant}

In this section we make some comments on the discriminant (2). See [4] for more details ([4] is strongly recommended).

For the equations

$$
f(x)=a x^{3}+b x^{2}+c x+d, \quad f^{\prime}(x)=3 a x^{2}+2 b x+c
$$

the resultant $R\left(f, f^{\prime}\right)$ of $f$ and $f^{\prime}$ is given by

$$
R\left(f, f^{\prime}\right)=\left|\begin{array}{ccccc}
a & b & c & d & 0 \\
0 & a & b & c & d \\
3 a & 2 b & c & 0 & 0 \\
0 & 3 a & 2 b & c & 0 \\
0 & 0 & 3 a & 2 b & c
\end{array}\right|
$$

It is easy to calculate (22) and the result becomes

$$
\frac{1}{a} R\left(f, f^{\prime}\right)=27 a^{2} d^{2}+4 a c^{3}-18 a b c d-b^{2} c^{2}+4 b^{3} d=-D .
$$

On the other hand, if $\alpha, \beta, \gamma$ are three solutions of $f(x)=0$ in $(21)$, then the following relations are well-known

$$
\alpha+\beta+\gamma=-\frac{b}{a}, \quad \alpha \beta+\alpha \gamma+\beta \gamma=\frac{c}{a}, \quad \alpha \beta \gamma=-\frac{d}{a} .
$$

From these it is easy to see

$$
\begin{aligned}
& \alpha+\beta+\gamma=-\frac{b}{a}, \quad \alpha^{2}+\beta^{2}+\gamma^{2}=\frac{b^{2}-2 a c}{a^{2}}, \quad \alpha^{3}+\beta^{3}+\gamma^{3}=-\frac{b^{3}+3 a^{2} d-3 a b c}{a^{3}} \\
& \alpha^{4}+\beta^{4}+\gamma^{4}=\frac{b^{4}+4 a^{2} b d+2 a^{2} c^{2}-4 a b^{2} c}{a^{4}}
\end{aligned}
$$

\footnotetext{
${ }^{1}$ The author owes the calculation to Hiroshi Oike.
} 
If we set

$$
\Delta=(\alpha-\beta)(\alpha-\gamma)(\beta-\gamma)
$$

the discriminant $D$ is given by

$$
D=a^{4} \Delta^{2} .
$$

Let us calculate $\Delta^{2}$ directly. For the Vandermonde matrix

$$
V=\left(\begin{array}{ccc}
1 & 1 & 1 \\
\alpha & \beta & \gamma \\
\alpha^{2} & \beta^{2} & \gamma^{2}
\end{array}\right) \Longrightarrow|V|=-\Delta
$$

we obtain by some manipulations of determinant

$$
\begin{aligned}
& \Delta^{2}=(-|V|)^{2}=|V|\left|V^{T}\right|=\left|V V^{T}\right|=\left|\begin{array}{ccc}
3 & \alpha+\beta+\gamma & \alpha^{2}+\beta^{2}+\gamma^{2} \\
\alpha+\beta+\gamma & \alpha^{2}+\beta^{2}+\gamma^{2} & \alpha^{3}+\beta^{3}+\gamma^{3} \\
\alpha^{2}+\beta^{2}+\gamma^{2} & \alpha^{3}+\beta^{3}+\gamma^{3} & \alpha^{4}+\beta^{4}+\gamma^{4}
\end{array}\right| \\
& =\left|\begin{array}{ccc}
3 & -\frac{b}{a} & \frac{b^{2}-2 a c}{a^{2}} \\
-\frac{b}{a} & \frac{b^{2}-2 a c}{a^{2}} & -\frac{b^{3}+3 a^{2} d-3 a b c}{a^{3}} \\
\frac{b^{2}-2 a c}{a^{2}} & -\frac{b^{3}+3 a^{2} d-3 a b c}{a^{3}} & \frac{b^{4}+4 a^{2} b d+2 a^{2} c^{2}-4 a b^{2} c}{a^{4}}
\end{array}\right| \\
& =\left|\begin{array}{ccc}
3 & -\frac{b}{a} & \frac{b^{2}-2 a c}{a^{2}} \\
\frac{2 b}{a} & -\frac{2 c}{a} & -\frac{3 a d-b c}{a^{2}} \\
-\frac{2 b^{2}+2 a c}{a^{2}} & -\frac{3 a d-3 b c}{a^{2}} & \frac{4 a b d+2 a c^{2}-2 b^{2} c}{a^{3}}
\end{array}\right| \\
& =\left|\begin{array}{ccc}
3 & -\frac{b}{a} & \frac{b^{2}-2 a c}{a^{2}} \\
\frac{2 b}{a} & -\frac{2 c}{a} & -\frac{3 a d-b c}{a^{2}} \\
-\frac{2 c}{a} & -\frac{3 a d-b c}{a^{2}} & \frac{a b d+2 a c^{2}-b^{2} c}{a^{3}}
\end{array}\right|=\left|\begin{array}{ccc}
3 & -\frac{b}{a} & -\frac{2 c}{a} \\
\frac{2 b}{a} & -\frac{2 c}{a} & -\frac{3 a d+b c}{a^{2}} \\
-\frac{2 c}{a} & -\frac{3 a d-b c}{a^{2}} & \frac{-2 b d+2 c^{2}}{a^{2}}
\end{array}\right| \\
& =\left|\begin{array}{ccc}
3 & -\frac{b}{a} & -\frac{2 c}{a} \\
0 & 2 \frac{b^{2}-3 a c}{3 a^{2}} & \frac{b c-9 a d}{3 a^{2}} \\
0 & \frac{b c-9 a d}{3 a^{2}} & 2 \frac{c^{2}-3 b d}{3 a^{2}}
\end{array}\right|=3\left|\begin{array}{cc}
2 \frac{b^{2}-3 a c}{3 a^{2}} & \frac{b c-9 a d}{3 a^{2}} \\
\frac{b c-9 a d}{3 a^{2}} & 2 \frac{c^{2}-3 b d}{3 a^{2}}
\end{array}\right| \\
& =\frac{1}{a^{4}} \frac{-1}{3}\left\{(b c-9 a d)^{2}-4\left(b^{2}-3 a c\right)\left(c^{2}-3 b d\right)\right\} .
\end{aligned}
$$

This result is very suggestive. In fact, from the cubic equation

$$
a x^{3}+b x^{2}+c x+d=0
$$

we have three data

$$
A=b^{2}-3 a c, \quad B=b c-9 a d, \quad C=c^{2}-3 b d,
$$


and so if we consider the quadratic equation

$$
A X^{2}+B X+C=0
$$

then the discriminant is just $B^{2}-4 A C$. This is very interesting.

Problem. Clarify the above connection.

As a result we have

$$
D=\frac{-1}{3}\left\{(b c-9 a d)^{2}-4\left(b^{2}-3 a c\right)\left(c^{2}-3 b d\right)\right\}=b^{2} c^{2}+18 a b c d-4 a c^{3}-4 b^{3} d-27 a^{2} d^{2} .
$$

\section{Some calculations}

In this section we calculate some quantities coming from the integral.

The expectation value $\left\langle x^{3}\right\rangle$ is formally given by

$$
\left\langle x^{3}\right\rangle=\frac{\iint x^{3} e^{-\left(a x^{3}+b x^{2} y+c x y^{2}+d y^{3}\right)} d x d y}{\iint e^{-\left(a x^{3}+b x^{2} y+c x y^{2}+d y^{3}\right)} d x d y}=-\frac{\partial}{\partial a} \log \left\{\iint e^{-\left(a x^{3}+b x^{2} y+c x y^{2}+d y^{3}\right)} d x d y\right\},
$$

so renormalized expectation values $\left\langle x^{3}\right\rangle_{\mathrm{RN}},\left\langle x^{2} y\right\rangle_{\mathrm{RN}},\left\langle x y^{2}\right\rangle_{\mathrm{RN}},\left\langle y^{3}\right\rangle_{\mathrm{RN}}$ are defined as

\section{Definition 2.}

$$
\begin{aligned}
& \left\langle x^{3}\right\rangle_{\mathrm{RN}}=-\frac{\partial}{\partial a} \log \left\{\ddagger \iint_{\mathbb{R}^{2}} e^{-\left(a x^{3}+b x^{2} y+c x y^{2}+d y^{3}\right)} d x d y \ddagger\right\}, \\
& \left\langle x^{2} y\right\rangle_{\mathrm{RN}}=-\frac{\partial}{\partial b} \log \left\{\ddagger \iint_{\mathbb{R}^{2}} e^{-\left(a x^{3}+b x^{2} y+c x y^{2}+d y^{3}\right)} d x d y \ddagger\right\}, \\
& \left\langle x y^{2}\right\rangle_{\mathrm{RN}}=-\frac{\partial}{\partial c} \log \left\{\ddagger \iint_{\mathbb{R}^{2}} e^{-\left(a x^{3}+b x^{2} y+c x y^{2}+d y^{3}\right)} d x d y \ddagger\right\}, \\
& \left\langle y^{3}\right\rangle_{\mathrm{RN}}=-\frac{\partial}{\partial d} \log \left\{\ddagger \iint_{\mathbb{R}^{2}} e^{-\left(a x^{3}+b x^{2} y+c x y^{2}+d y^{3}\right)} d x d y \ddagger\right\} .
\end{aligned}
$$

From the integral forms (5) and (6) it is easy to calculate the above. Namely, we have

$$
\begin{array}{ll}
\left\langle x^{3}\right\rangle_{\mathrm{RN}}=\frac{18 b c d-4 c^{3}-54 a d^{2}}{6 D}, & \left\langle x^{2} y\right\rangle_{\mathrm{RN}}=\frac{2 b c^{2}+18 a c d-12 b^{2} d}{6 D}, \\
\left\langle x y^{2}\right\rangle_{\mathrm{RN}}=\frac{2 b^{2} c+18 a b d-12 a c^{2}}{6 D}, & \left\langle y^{3}\right\rangle_{\mathrm{RN}}=\frac{18 a b c-4 b^{3}-54 a^{2} d}{6 D},
\end{array}
$$

where $D=b^{2} c^{2}+18 a b c d-4 a c^{3}-4 b^{3} d-27 a^{2} d^{2}$.

We can calculate other quantities like $\left\langle x^{5} y\right\rangle_{\mathrm{RN}}$ or $\left\langle x^{4} y^{2}\right\rangle_{\mathrm{RN}}$ by use of these ones, which will be left to readers.

\section{Concluding remarks}

In this paper we calculated the non-Gaussian integral (1) in a direct manner and, moreover, calculated some renormalized expectation values. It is not clear at the present time whether these results are useful enough or not. It would be desirable to accumulate many supporting evidences. Some application(s) will be reported elsewhere [5]. 
At this stage we can consider a further generalization. Namely, for the general degree $n$ polynomial

$$
f(x)=a_{0} x^{n}+a_{1} x^{n-1}+\cdots+a_{n-1} x+a_{n}
$$

the (non-Gaussian) integral becomes

$$
\int_{\mathbb{R}} \frac{1}{\sqrt[n]{f(x)^{2}}} d x
$$

The discriminant $D$ of the equation $f(x)=0$ is given by the resultant $R\left(f, f^{\prime}\right)$ of $f$ and $f^{\prime}$ like

$$
\frac{1}{a_{0}} R\left(f, f^{\prime}\right)=(-1)^{\frac{n(n-1)}{2}} D \Longleftrightarrow D=(-1)^{\frac{n(n-1)}{2}} R\left(f, f^{\prime}\right) / a_{0}
$$

where

$$
R\left(f, f^{\prime}\right)=\left|\begin{array}{cccccccc}
a_{0} & a_{1} & \cdots & a_{n-1} & a_{n} & & & \\
& a_{0} & a_{1} & \cdots & a_{n-1} & a_{n} & & \\
& & \ddots & & & \ddots & & \\
& & & a_{0} & a_{1} & \cdots & a_{n-1} & a_{n} \\
n a_{0} & (n-1) a_{1} & \ldots & a_{n-1} & & & & \\
& n a_{0} & (n-1) a_{1} & \cdots & a_{n-1} & & & \\
& & \ddots & & & \ddots & & \\
& & & & n a_{0} & (n-1) a_{1} & \cdots & a_{n-1}
\end{array}\right|,
$$

see $(21)$ and (22).

For example, for $n=4$

$$
R\left(f, f^{\prime}\right)=\left|\begin{array}{ccccccc}
a_{0} & a_{1} & a_{2} & a_{3} & a_{4} & 0 & 0 \\
0 & a_{0} & a_{1} & a_{2} & a_{3} & a_{4} & 0 \\
0 & 0 & a_{0} & a_{1} & a_{2} & a_{3} & a_{4} \\
4 a_{0} & 3 a_{1} & 2 a_{2} & a_{3} & 0 & 0 & 0 \\
0 & 4 a_{0} & 3 a_{1} & 2 a_{2} & a_{3} & 0 & 0 \\
0 & 0 & 4 a_{0} & 3 a_{1} & 2 a_{2} & a_{3} & 0 \\
0 & 0 & 0 & 4 a_{0} & 3 a_{1} & 2 a_{2} & a_{3}
\end{array}\right|
$$

and

$$
\begin{aligned}
D= & 256 a_{0}^{3} a_{4}^{3}-4 a_{1}^{3} a_{3}^{3}-27 a_{0}^{2} a_{3}^{4}-27 a_{1}^{4} a_{4}^{2}-128 a_{0}^{2} a_{2}^{2} a_{4}^{2}+a_{1}^{2} a_{2}^{2} a_{3}^{2}+16 a_{0} a_{2}^{4} a_{4} \\
& -4 a_{0} a_{2}^{3} a_{3}^{2}-4 a_{1}^{2} a_{2}^{3} a_{4}+144 a_{0}^{2} a_{2} a_{3}^{2} a_{4}-6 a_{0} a_{1}^{2} a_{3}^{2} a_{4}+144 a_{0} a_{1}^{2} a_{2} a_{4}^{2}-192 a_{0}^{2} a_{1} a_{3} a_{4}^{2} \\
& +18 a_{0} a_{1} a_{2} a_{3}^{3}+18 a_{1}^{3} a_{2} a_{3} a_{4}-80 a_{0} a_{1} a_{2}^{2} a_{3} a_{4},
\end{aligned}
$$

and for $n=5$

$$
R\left(f, f^{\prime}\right)=\left|\begin{array}{ccccccccc}
a_{0} & a_{1} & a_{2} & a_{3} & a_{4} & a_{5} & 0 & 0 & 0 \\
0 & a_{0} & a_{1} & a_{2} & a_{3} & a_{4} & a_{5} & 0 & 0 \\
0 & 0 & a_{0} & a_{1} & a_{2} & a_{3} & a_{4} & a_{5} & 0 \\
0 & 0 & 0 & a_{0} & a_{1} & a_{2} & a_{3} & a_{4} & a_{5} \\
5 a_{0} & 4 a_{1} & 3 a_{2} & 2 a_{3} & a_{4} & 0 & 0 & 0 & 0 \\
0 & 5 a_{0} & 4 a_{1} & 3 a_{2} & 2 a_{3} & a_{4} & 0 & 0 & 0 \\
0 & 0 & 5 a_{0} & 4 a_{1} & 3 a_{2} & 2 a_{3} & a_{4} & 0 & 0 \\
0 & 0 & 0 & 5 a_{0} & 4 a_{1} & 3 a_{2} & 2 a_{3} & a_{4} & 0 \\
0 & 0 & 0 & 0 & 5 a_{0} & 4 a_{1} & 3 a_{2} & 2 a_{3} & a_{4}
\end{array}\right|
$$


and

$$
\begin{aligned}
& D=3125 a_{0}^{4} a_{5}^{4}-2500 a_{0}^{3} a_{1} a_{4} a_{5}^{3}-3750 a_{0}^{3} a_{2} a_{3} a_{5}^{3}+2000 a_{0}^{3} a_{2} a_{4}^{2} a_{5}^{2}+2250 a_{0}^{3} a_{3}^{2} a_{4} a_{5}^{2} \\
& -1600 a_{0}^{3} a_{3} a_{4}^{3} a_{5}+256 a_{0}^{3} a_{4}^{5}+2000 a_{0}^{2} a_{1}^{2} a_{3} a_{5}^{3}-50 a_{0}^{2} a_{1}^{2} a_{4}^{2} a_{5}^{2}+2250 a_{0}^{2} a_{1} a_{2}^{2} a_{5}^{3} \\
& -2050 a_{0}^{2} a_{1} a_{2} a_{3} a_{4} a_{5}^{2}+160 a_{0}^{2} a_{1} a_{2} a_{4}^{3} a_{5}-900 a_{0}^{2} a_{1} a_{3}^{3} a_{5}^{2}+1020 a_{0}^{2} a_{1} a_{3}^{2} a_{4}^{2} a_{5} \\
& -192 a_{0}^{2} a_{1} a_{3} a_{4}^{4}-900 a_{0}^{2} a_{2}^{3} a_{4} a_{5}^{2}+825 a_{0}^{2} a_{2}^{2} a_{3}^{2} a_{5}^{2}+560 a_{0}^{2} a_{2}^{2} a_{3} a_{4}^{2} a_{5}-128 a_{0}^{2} a_{2}^{2} a_{4}^{4} \\
& -630 a_{0}^{2} a_{2} a_{3}^{3} a_{4} a_{5}+144 a_{0}^{2} a_{2} a_{3}^{2} a_{4}^{3}+108 a_{0}^{2} a_{3}^{5} a_{5}-27 a_{0}^{2} a_{3}^{4} a_{4}^{2}-1600 a_{0} a_{1}^{3} a_{2} a_{5}^{3} \\
& +160 a_{0} a_{1}^{3} a_{3} a_{4} a_{5}^{2}-36 a_{0} a_{1}^{3} a_{4}^{3} a_{5}+1020 a_{0} a_{1}^{2} a_{2}^{2} a_{4} a_{5}^{2}+560 a_{0} a_{1}^{2} a_{2} a_{3}^{2} a_{5}^{2}-746 a_{0} a_{1}^{2} a_{2} a_{3} a_{4}^{2} a_{5} \\
& +144 a_{0} a_{1}^{2} a_{2} a_{4}^{4}+24 a_{0} a_{1}^{2} a_{3}^{3} a_{4} a_{5}-6 a_{0} a_{1}^{2} a_{3}^{2} a_{4}^{3}-630 a_{0} a_{1} a_{2}^{3} a_{3} a_{5}^{2}+24 a_{0} a_{1} a_{2}^{3} a_{4}^{2} a_{5} \\
& +356 a_{0} a_{1} a_{2}^{2} a_{3}^{2} a_{4} a_{5}-80 a_{0} a_{1} a_{2}^{2} a_{3} a_{4}^{3}-72 a_{0} a_{1} a_{2} a_{3}^{4} a_{5}+18 a_{0} a_{1} a_{2} a_{3}^{3} a_{4}^{2}+108 a_{0} a_{2}^{5} a_{5}^{2} \\
& -72 a_{0} a_{2}^{4} a_{3} a_{4} a_{5}+16 a_{0} a_{2}^{4} a_{4}^{3}+16 a_{0} a_{2}^{3} a_{3}^{3} a_{5}-4 a_{0} a_{2}^{3} a_{3}^{2} a_{4}^{2}+256 a_{1}^{5} a_{5}^{3}-192 a_{1}^{4} a_{2} a_{4} a_{5}^{2} \\
& -128 a_{1}^{4} a_{3}^{2} a_{5}^{2}+144 a_{1}^{4} a_{3} a_{4}^{2} a_{5}-27 a_{1}^{4} a_{4}^{4}+144 a_{1}^{3} a_{2}^{2} a_{3} a_{5}^{2}-6 a_{1}^{3} a_{2}^{2} a_{4}^{2} a_{5}-80 a_{1}^{3} a_{2} a_{3}^{2} a_{4} a_{5} \\
& +18 a_{1}^{3} a_{2} a_{3} a_{4}^{3}+16 a_{1}^{3} a_{3}^{4} a_{5}-4 a_{1}^{3} a_{3}^{3} a_{4}^{2}-27 a_{1}^{2} a_{2}^{4} a_{5}^{2}+18 a_{1}^{2} a_{2}^{3} a_{3} a_{4} a_{5}-4 a_{1}^{2} a_{2}^{3} a_{4}^{3} \\
& -4 a_{1}^{2} a_{2}^{2} a_{3}^{3} a_{5}+a_{1}^{2} a_{2}^{2} a_{3}^{2} a_{4}^{2} \text {. }
\end{aligned}
$$

However, to write down the general case explicitly is not easy (almost impossible).

Problem. Calculate (23) for $n=4$ (and $n=5)$ directly.

The wall called Gaussian is very high and not easy to overcome, and therefore hard work will be needed.

Recently the subsequent paper [6] by Morozov and Shakirov appeared. Our works are deeply related to so-called non-linear algebras, so we will make some comments on this point in a near future. As a general introduction to them see for example [7].

The author thanks referees and Hiroshi Oike, Ryu Sasaki for many useful suggestions and comments.

\section{References}

[1] Fujii K., Beyond Gaussian: a comment, arXiv:0905.1363.

[2] Morozov A., Shakirov Sh., Introduction to integral discriminants, J. High Energy Phys. 2009 (2009), no. 12, 002, 39 pages, arXiv:0903.2595.

[3] Whittaker E.T., Watson G.N., A course of modern analysis, Cambridge University Press, Cambridge, 1996.

[4] Satake I., Linear algebra, Shokabo, Tokyo, 1989 (in Japanese).

[5] Fujii K., Beyond the Gaussian. II. Some applications, in progress.

[6] Morozov A., Shakirov Sh., New and old results in resultant theory, Theoret. and Math. Phys. 163 (2010), 587-617, arXiv:0911.5278.

[7] Dolotin V., Morozov A., Introduction to non-linear algebra, World Scientific Publishing Co. Pte. Ltd., Hackensack, NJ, 2007, hep-th/0609022. 